\title{
Complex form landscape of a chiral solid-solution: where does one form end and the next begin?
}

Kevin J. Gagnon ${ }^{\mathrm{a}}$, Jicong $\mathrm{Li}^{\mathrm{a}}$, Ales Medek ${ }^{\mathrm{a}}$, Mettachit Navamal ${ }^{\mathrm{a}}$, Helen $\mathrm{Shi}^{\mathrm{a}}$, and Sonja Sharpe ${ }^{a}$

a. Vertex Pharmaceuticals Inc. 50 Northern Avenue, Boston, Massachusetts 02210, USA.

\section{Email Contact: Kevin_Gagnon@vrtx.com}

The separation of enantiomers is of great interest to the pharmaceutical industry. One of the tools utilized for the production of a pure chiral active pharmaceutical ingredient (API) is enrichment of enantiomers by crystallization. Resolution of a chiral compound strongly depends on the kind of solid state crystal lattice that it forms. Three common types of racemates include racemic compound, conglomerate and solid solution.

Thorough solid state characterizations of (S)-3,5,5-trimethylpyrrolidin-2-one is presented. While the solution phase suggested a simple racemic solid solution, the solid phase proved to be much more complex. Multiple solid phases exist; however, the system still seems to be on a solid solution continuum. Figure 1. shows the unique characteristics of the solubility curve vs chiral purity overlayed with cartoon representations of the complex phases.

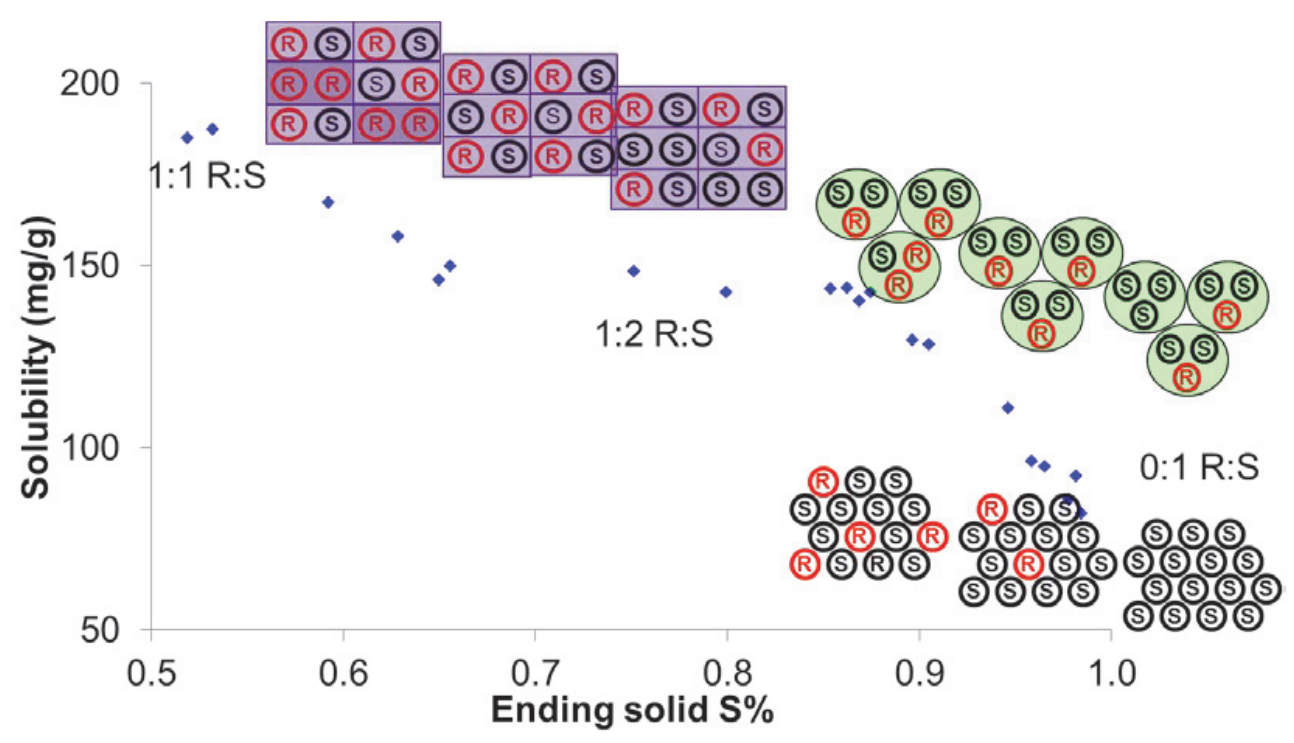

Figure 1. Solubility curve vs. chiral solid ratio for 3,5,5-trimethylpyrrolidin-2-one showing cartoon schematics of the identified forms. 